\title{
Un ejemplo de dinámica de role playing en una asignatura de libre configuración en Derecho
}

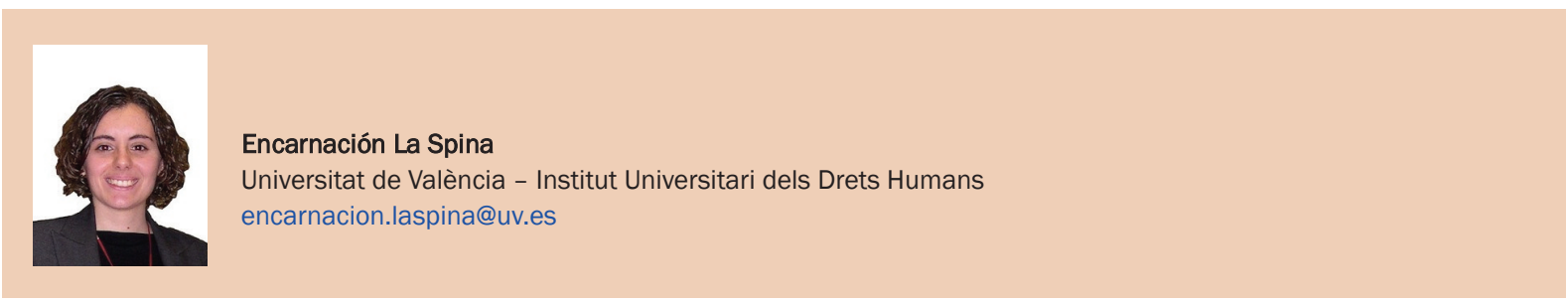

|Fecha presentación: 14/02/2011 | Aceptación: 10/05/2011 |Publicación: 21/06/2011

\section{Resumen}

Las exigencias del actual mundo laboral y profesional requieren una mayor creación de espacios de formación universitaria para la solución de problemas reales. El role playing, como técnica didáctica diseñada para asignaturas de libre configuración cursadas por estudiantes de diferente perfil formativo, facilita el proceso de enseñanza-aprendizaje de contenidos complejos del Derecho. Este artículo trata de describir y evaluar la experiencia docente en una dinámica basada en la simulación para la asignatura "Convención sobre la eliminación de todas las formas de discriminación contra la mujer: la singularidad del Mediterráneo".

Palabras clave: role playing, Derecho, estudiantes, docencia, aprendizaje activo

\begin{abstract}
Resum
Les exigències del actual món laboral i professional demanen una major creació de espais de formació universitària per a la solució de problemes reals. El role playing, com a tècnica didàctica dissenyada per a assignatures de lliure configuració cursades per estudiants de diferent perfil formatiu, facilita el procés d' ensenyament- aprenentatge de continguts complexos del Dret. Aquest article tracta de descriure i avaluar l' experiència docent en una dinàmica basada en la simulació per l' assignatura “Convenció sobre l' eliminació de totes les formes de discriminació contra la dona: la singularitat del Mediterràni".
\end{abstract}

Paraules Clau: role playing, Dret, estudiants, docència, aprenentatge actiu

\section{Abstract}

The current labour and professional world requires a major creation of spaces of university education to solve real problems. The role playing, as didactic tool to free choice subjects studied by students of different training specification, facilitates the formative process of complex contents of the Law. This paper tries to describe and evaluate the educational experience in a dynamic based in the role playing for the subject "Convention on the elimination of all the forms of discrimination against the woman: the singularity of Mediterranean ".

Keywords: role playing, Law, students, teaching, active learning 


\section{Introducción}

Actualmente, el Espacio Europeo de Educación Superior implica proporcionar una formación universitaria en la que se integren armónicamente las competencias genéricas básicas, las competencias transversales relacionadas con la formación integral de las personas y las competencias más específicas que posibiliten una mayor orientación e integración profesional en el mercado de trabajo (Marcelo 1996: 7). La creación de espacios de formación universitaria que cumplan con la misión de preparar para la solución de problemas o contenidos prácticos puede hacer realmente efectivos los procesos de aprendizaje en la medida en que permite probar, construir, experimentar, tomar decisiones y ser más resolutivos (Barkley, Cross y Major 2006: 20).

Entre las técnicas y posibilidades de innovación educativa existentes y las variantes metodológicas en el modelo de enseñanza-aprendizaje universitario (Català 2007), viene siendo recurrente la aplicación del role playing en el diseño de las dinámicas de habilidades jurídicas fundamentales (García Añón Roig et al. 2008) y en asignaturas de libre configuración del área de conocimiento de Filosofía del Derecho, Moral y Política de la Facultat de Dret de la Universitat de València (García Añón 2009). De hecho, este aprendizaje activo de trabajo en grupo basado en el estudio, análisis, desarrollo y resolución de situaciones reales, ha sido implementado y consolidado satisfactoriamente en "Problemas actuales de Teoría y Filosofía Política"1 ${ }^{\text {" }}$ otra oferta académica de la misma área de conocimiento. En este trabajo se tratará de presentar una dinámica diferente de juego de roles diseñada para asignaturas de libre elección habitualmente impartidas en la modalidad de jornadas formativas o cursos con menor volumen o carga de trabajo respecto a los módulos troncales. En este caso, "Convención sobre la eliminación de toda discriminación contra la mujer: la singularidad del Mediterráneo"2, es un módulo de libre elección de 2 créditos, de carácter teórico-práctico, que plantea combinar el estudio y comentario de textos, la jurisprudencia con el visionado de películas y, en el curso 2008/2009, la simulación de casos. De este modo, en última instancia se quiere poner de manifiesto como una metodología basada en los principios del aprendizaje colaborativo puede ser un eficaz complemento educativo para mejorar (Parcerisa 2010: 74) y fomentar el interés en contenidos complejos del Derecho (resolución judicial y argumentación jurídica) para estudiantes con disímil perfil formativo.

\section{Objetivos}

El trabajo realizado en esta investigación educativa tiene como objetivo principal realizar una aportación al desarrollo de metodologías coherentes con las exigencias del Espacio Europeo de Educación Superior. Para ello, se ha experimentado una metodología activa basada en los objetivos del aprendizaje colaborativo (Johnson y Smith 1998: 21-23; Ovejero 1990) para analizar la utilidad del role playing en el logro de la interdependencia positiva, la interacción pro- motora, la responsabilidad individual y de grupo, el desarrollo de las competencias de trabajo en equipo y la valoración del grupo en un contexto marcado por la heterogeneidad. De hecho, la "Convención sobre la eliminación de todas las formas de discriminación contra la mujer: la singularidad del Mediterráneo" ha sido y es cursada habitualmente por estudiantes inscritos a otras disciplinas del área de ciencias sociales y humanidades que podrían tener dificultades a la hora de desarrollar los siguientes objetivos específicos de la asignatura:

- Proporcionar herramientas conceptuales que permitan analizar y reflexionar sobre los mecanismos de protección contra las diferentes formas de discriminación de la mujer.

- Conocer el funcionamiento y los principales mecanismos de protección de la Convención sobre la eliminación de todas las formas de discriminación contra la mujer y el Protocolo adicional de la CEDAW.

- Contribuir al debate y la argumentación sobre cuestiones concretas: derechos políticos y movimientos de mujeres, igualdad y no discriminación, diversidad cultural y derechos de las mujeres desde una perspectiva de género, combinando ejercicios teórico-prácticos.

- Aprender a interpretar y discutir contenidos incluidos en resoluciones judiciales para resolver y proponer soluciones a casos o problemas concretos.

El perfil formativo de los estudiantes de la asignatura el curso 2008/2009, de acuerdo a los datos disponibles en la plataforma de Aula Virtual de la Universitat de València era ciertamente muy heterogéneo: un 63,15\% de estudiantes cursaba la licenciatura en Derecho; un 18,42\% Relaciones Laborales; un 5,26\% Ciencias Empresariales; un 5,26\% Economía; un 2,63\% Farmacia; un 2,63\% Pedagogía; y un 2,63 \% Ciencias del Trabajo. Por tanto, si bien había una mayoría de estudiantes de formación jurídica, un $37 \%$ eran prácticamente legos en Derecho.

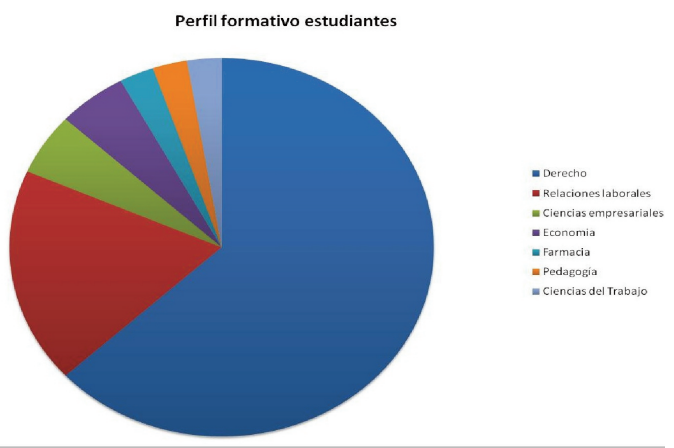

Figura 1: Perfil formativo estudiantes.

Por este motivo, este elemento de heterogeneidad en la formación de grupos de aprendizaje colaborativo, era un aspecto importante a considerar a la hora de estructurar e impartir

\footnotetext{
${ }^{1}$ La asignatura de 4,5 créditos, “Problemas actuales de Teoría y Filosofía Política”, se imparte desde el curso 2003-2004 por los profesores Javier De Lucas y José García Añón. En este caso, se trataba de implicar la formación de grupos que simularán ser el equipo de asesores de la campaña electoral de un líder político para responder y resolver desde cada teoría política los problemas que les plantea la actualidad. Puede consultarse el trabajo realizado en el blog de la asignatura : http://spina.blogs.uv.es/

2 La asignatura de 2 créditos "Convención sobre la eliminación de toda discriminación contra la mujer: la singularidad del Mediterráneo" se impartía el curso 2008/2009 por las profesoras Cristina García Pascual, Ruth Mestre i Mestre, María José Añón Roig y Ángeles Solanes Corella, y durante ese curso académico he asumido íntegramente la colaboración docente de esta asignatura.
} 
unos contenidos complejos del Derecho y, de igual modo, en esta investigación evaluativa, su alcance revestía un potencial interesante para medir el grado de implicación (Martín 1992: 78) y el éxito innovador de la metodología escogida.

\section{Diseño del método de enseñanza y procedi- miento}

El diseño de metodologías de aprendizaje exige la integración de tres componentes fundamentales: cómo organizar los aprendizajes de los alumnos, cómo desarrollar dichos aprendizajes y cómo evaluarlos. Esto es, según De Miguel (2006: 17-18) las modalidades de enseñanza, los métodos y las estrategias evaluativas. El profesor universitario dispone de libertad para diseñar los métodos que personalizan en cierto modo el proceso de enseñanza-aprendizaje de la asignatura (Bain 2006: 5). En este caso, la aplicación del role playing en curso prefijado e integrado en la oferta formativa de los planes de estudios universitarios para la libre configuración del currículum del estudiante permite aportar fácilmente mayor participación y autonomía al estudiante por medio de una reducción de las horas presenciales (Labrador et al. 2008: 71). Y, de igual modo permite introducir situaciones o problemas actuales que les motivaran a utilizar estrategias de búsqueda de información, aplicar nuevos conocimientos para la solución de problemas jurídicos, tomar decisiones y trabajar de forma autónoma, reflexiva y crítica.

Para el desarrollo de esta investigación educativa se han planteado unas primeras sesiones de presentación sobre el marco teórico y la significación de la Convención sobre la eliminación de todas las forma de discriminación contra la mujer, por medio del visionado de películas y entrega de cuestionarios en la línea de anteriores ediciones de la asignatura. Así, una vez superada la iniciación teórica de las primeras sesiones, era posible evaluar el desarrollo integrado de conocimientos, habilidades y valores en el aprendizaje basado en problemas por medio de una dinámica final de simulación o juego de roles de un caso ante el Comité de la eliminación de todas las formas de discriminación contra la mujer, que es un órgano que puede recibir y examinar las denuncias por violaciones a los derechos protegidos por la Convención. $\mathrm{Al}$ efecto, los estudiantes se han distribuido en tres grupos de 10 alumnos aproximadamente que representan el rol de: Estado, ONG y Comité CEDAW. En el grupo se nombra, al menos, un coordinador/a (se encarga de realizar la distribución de tareas, supervisión, verificar el trabajo final, etc.); un portavoz (realizará la exposición final en la que se expondrán los argumentos de la parte); y un secretario/a (elaborará un informe de las tareas realizadas, fechas de reunión, duración, persona encargada...). Los grupos no están formados desde el inicio del curso sino que se concede libertad de elección a los estudiantes de acuerdo a sus preferencias, salvo una cuota de equilibrio de perfiles formativos jurídicos y no en cada grupo y subgrupo, según los principios del aprendizaje colaborativo.

Por este motivo, y habida cuenta de la heterogeneidad formativa de los estudiantes, el diseño de la dinámica sigue una estructura sencilla y adaptada por el profesor en la selección de los materiales de trabajo básicos y complementarios acompañados de una guía de la actividad integrada por el contenido adaptado de una sentencia del Tribunal Euro- peo de Derechos Humanos y el voto disidente ${ }^{3}$, así como la simplificación de los hechos a valorar, que son comunes para los subgrupos y grupos:

\section{Hechos}

Las demandantes nacieron en 1973 y viven en Viena desde 1999, año en el que abandonaron su país para seguir sus estudios de medicina en la Facultad de Medicina de la Universidad de esa ciudad. El 26 de agosto de 1997, las demandantes se matricularon en la Facultad de Medicina, llevando el velo islámico durante los cuatro años de estudios de medicina en la Universidad de Bursa y posteriormente, hasta febrero de 1998. El Rector de la Universidad de Estambul difundió una circular reglamentando la entrada de los estudiantes en el campus universitario. La parte aplicable de esta circular dice:

"En virtud de la Constitución, de la legislación, de los reglamentos, $y$ de conformidad con la jurisprudencia del Consejo de Estado, de la Comisión europea de Derechos Humanos y las decisiones adoptadas por los comités administrativos de las universidades, las estudiantes que lleven la "cabeza cubierta" (llevando el velo islámico) y los estudiantes que lleven barba (incluidos los estudiantes extranjeros) no deben ser admitidos en los cursos, cursillos $y$ prácticas. En consecuencia, el nombre y el número de las estudiantes que lleven el velo islámico o de los estudiantes con barba no deben constar en las listas de estudiantes". Conforme a esta circular, el 12 de marzo de 1998, los vigilantes negaron a las demandantes el acceso a las pruebas escritas del curso de oncología porque llevaba el velo islámico. En mayo de 1998 se inició un procedimiento disciplinario contra las demandantes en virtud del artículo 6.a) del Reglamento sobre Procedimientos Disciplinarios de los Estudiantes. Las demandantes han agotado la vía interna y han acudido a una ONG para presentar una denuncia individual ante el Comité CEDAW según lo dispuesto en el Protocolo adicional de la CEDAW.

En base a estos hechos, se determinan tres ejes de discusión que deben extraer del texto los estudiantes para poder responder a una serie de cuestiones: los derechos protegidos por la Convención y los argumentos de la sentencia relacionados con la posición defendida, relacionándolos con los hechos expuestos. Se plantea así una reelaboración y argumentación por cada grupo para justificar la interpretación más adecuada a la posición defendida, que en última instancia deberá ser valorada por el Comité CEDAW. Para ello, se deben seleccionar y exponer los argumentos de cada parte recogidos en los fundamentos jurídicos sobre la significación del uso del velo, la igualdad de las mujeres y el derecho a la educación. De este modo, la lectura conjunta del principal material de trabajo, la sentencia, se adapta y trabaja en grupo para que los estudiantes con perfiles formativos no jurídicos indirectamente puedan dialogar, confrontar, tomar conciencia e implicarse en valores, hipótesis y opiniones de polémicas consecuencias, validando y complementando su conocimiento (Martín 1992: 78).

A tal propósito, junto a la adaptación del lenguaje jurídico, es necesario salvar la extensión de la resolución judicial con subdivisiones de los fundamentos jurídicos de las

${ }^{3}$ Sentencia del Tribunal Europeo de Derechos Humanos (Sección 4a). Caso Leyla Çahin contra Turquía. Sentencia de 29 junio 2004 TEDH $\backslash 2004 \backslash 46$. Se proponen algunas lecturas obligatorias: Mernissi (2002); Taylor (2000). 
partes y del voto disidente para formular la postura del Estado (parte demandada), ONG (parte demandante) y el Comité CEDAW (el órgano cuasi-judicial que emite recomendaciones y propuestas a las partes sobre el tema objeto de estudio).

De este modo, cada subgrupo elabora una guía de exposición, recogiendo observaciones y explicando los diferentes roles, como el modelo:

\begin{tabular}{|l|l|l|}
\hline Posición deIESTADO & $\begin{array}{l}\text { Posición de la ONG'S } \\
\text { Foularddroit.org }\end{array}$ & $\begin{array}{l}\text { Posición/Decisión del } \\
\text { Comité CEDAW }\end{array}$ \\
\hline Argumentos utilizados en & Argumentos utilizados en & Argumentos utilizados \\
fundamentos de Derecho. & fundamentos de Derecho. & en fallo y voto disidente. \\
\hline $1-$ & $1-$ & $1-$ \\
$2-$ & $2-$ & $2-$ \\
$3-$ & $3-$ & $3-$ \\
\hline Observaciones & Observaciones & Observaciones \\
\hline
\end{tabular}

Esta reestructuración orientada por el profesor, facilita a los estudiantes con un perfil formativo no jurídico aprehender los contenidos y conceptos jurídicos que deben defender desde cada rol. Desde el inicio del curso, los materiales y las indicaciones se trabajan en grupo y sólo en la última sesión presencial por medio del role playing se discute la postura defendida y los argumentos elaborados con la propuesta durante un periodo de 20 minutos con el grupo. Y, a posteriori se exponen ante el Comité CEDAW que modera el debate durante otros 30 minutos (esta simulación queda grabada). Una vez concluido el debate, el grupo que representa al Comité CEDAW, un órgano de vigilancia y aplicación de la Convención por los Estados que la hubieren ratificado o se hubieren adherido a ella, comunica la decisión favorable o desfavorable respecto de la denuncia individual presentada por violación de los derechos protegidos por la Convención.

Los estudiantes deben entregar en la plataforma de Aula Virtual de la Universitat de València por grupos la posición defendida que representan junto a un breve comentario y opinión o valoración personal sobre la dinámica propuesta De este modo, para la evaluación continua de las competencias adquiridas se secuencian los procedimientos y técnicas evaluativas del siguiente modo: durante el desarrollo de la actividad, por medio del seguimiento de las tareas individuales y del grupo durante las sesiones, y al final, a través de la participación, la exposición oral, la argumentación sostenida durante el debate por cada parte y el análisis del producto definitivo, así como de las opiniones personales u observaciones sobre las habilidades, competencias y conocimientos adquiridos en Derecho (Delgado 2006:34). Para ello, resulta de especial utilidad la grabación audiovisual de la sesión de la dinámica final, pues permite valorar al profesor y los estudiantes el desarrollo de la misma, así como autoevaluar en su visionado aquellos aspectos mejorables en el aula universitaria.

\section{Resultados y conclusión}

Entre los resultados positivos obtenidos, cabe centrarse en aquellos que han reportado la aplicación de la técnica del role playing respecto al estudiante y al profesor (Alfaro Rocher 2007). Esta metodología activa, participativa e interpersonal, permite acceder al conocimiento de forma significativa, pues convierte en relevantes informaciones que serían transmitidas y aprehendidas de modo meramente memorístico. De igual modo, permite desarrollar habilidades y destrezas para comunicarse en público, para estudiantes que en un cierto modo no están acostumbrados a exponer, extrapolar conceptos y argumentar sus ideas en un contexto interdisciplinar. Otro aporte del role playing es estimular una lectura comprensiva de los materiales y lecturas recomendadas, para preparar las intervenciones y trabajos propuestos. En definitiva, permite fomentar el potencial creativo e imaginativo de los estudiantes, haciendo trabajar el razonamiento y la lógica durante el transcurso de la simulación, además de plantear los retos y posibles confrontaciones existentes entre los grupos y cómo poder solucionarlos. La técnica del role playing en general, y su aplicación a esta asignatura en particular, permite desarrollar en los estudiantes la empatía y la tolerancia. Esto es así porque la técnica de la representación de diversos planteamientos, no necesariamente coincidentes con los defendidos por los estudiantes, ayuda a comprender qué significa meterse en la piel del otro, o incluso a plantearse qué sienten al defender situaciones que pueden ser ajenas en un principio a la propia ideología o área de conocimiento, pero presentes en nuestra sociedad. Son igualmente importantes la toma de conciencia y la responsabilidad con problemas actuales cuya resolución política es compleja, por lo que crean en el estudiante una consciencia más amplia y crítica de sus decisiones y de los problemas planteados. En definitiva, se trata de combinar la capacidad de trabajo en grupo y la toma de decisiones, habilidades que son de gran importancia en la sociedad y muy valoradas a nivel profesional (Ribas 2000: 3 ).

De este modo, como resultado de la combinación de los objetivos del proceso de enseñanza-aprendizaje activo y colaborativo, los estudiantes han podido desarrollar y adquirir las siguientes competencias (Dasí et al., 2007; De Miguel 2006, Aguaded-Gómez y Fonseca-Mora 2009: 60):

- Análisis y síntesis de la información de manera autónoma, usando fuentes diversas.

- Capacidad crítica constructiva, aportando su visión y enriqueciendo el resultado

- Mayor capacidad de comunicación oral y escrita, utilizando terminología jurídica de la asignatura.

- Apreciación de las dificultades y potencialidades del trabajo en equipo.

- Resolución de problemas y aplicación del conocimiento a la práctica.

Desde el punto de vista del profesor, la aplicación del role playing en esta experiencia docente ha permitido reforzar aspectos positivos de un tránsito de lo tradicional a lo emergente en el ámbito educativo. Por ejemplo, ha superado satisfactoriamente una relación jerárquica de saber con el refuerzo de la idea de su complejidad y el manejo de diversidad de saberes vinculados a un tema particular o a un perfil de estudiante necesariamente heterogéneo, como es nuestra realidad social. Y, en particular, pese al mayor volumen de trabajo que supone para el docente universitario aprender a innovar e innovar para aprender en el aula universitaria (Centre for Educational Research and innovation 2008: 10), su incorporación en asignaturas de libre configuración es igualmente positiva, en la medida en que el estudiante que habitualmente adopta un rol de sujeto pasivo en modalidades organizativas de aprendizaje meramente teórico deviene un sujeto de aprendizaje más activo o inevitablemente más implicado "porque se trata de sus impactos" (Martín 1992: 77-78). 


\section{Bibliografía}

Aguaded-Gómez, J. Ignacio; Fonseca-Mora, $\mathrm{M}^{\mathrm{a}}$ Carmen (Coord.) (2009). Huellas de innovación docente en las aulas universitarias. La Coruña: Netbiblo.

Alfaro Rocher, Ignacio Javier (Dir.) (2007): La respuesta organizativa y metodológica de la Universidad ante una nueva tipología de estudiantes. València: Publicacions de la Universitat de València.

Bain, Ken (2006). Lo que hacen los mejores profesores de Universidad .València: Publicacions de la Universitat de València.

Barkley, E.F.; Cross, K. Patricia; Major, C. Howell (2006). Técnicas de aprendizaje colaborativo: manual para el profesorado universitario. Madrid: Morata - Ministerio de Educación y Ciencia.

Català Sanz, Jorge Antonio (2007): Innovación educativa en la universidad: historia. València: Publicacions de la Universitat de València.

Centre for Educational Research and Innovation (2008): Innovating to Learn, Learning to Innovate. Paris: OECD.

Dasí Coscollà, Àngels et al. (2007). Innovación educativa en la Universidad: ADE-Derecho. València: Publicacions de la Universitat de València.

Delgado García, Ana M. (Coord.) (2006). Evaluación de las competencias en el Espacio Europeo de Educación Superior. Una experiencia desde el Derecho y la Ciencia Política. Barcelona: Bosch.

García Añón, José (Coord.) (2009). Miradas a la innovación: experiencias de innovación en la docencia del Derecho València: Servei de Formació Permanent, Universitat de València. http://issuu.com/josegarciaanon/docs/2009miradas

García Añón, José et al. (2008). "Diseño de materiales para el aprendizaje de habilidades jurídicas fundamentales," @tic: revista d'innovació educativa, 1, pp. 37-44. http://ojs.uv.es/index.php/attic/article/view/49/44

Johnson, Roger T.; Smith, Karl A. (1998): Active learning: Cooperation in the college classroom. Edina: Interaction Book Company.

Labrador Piquer, María José, Andreu Andrés, María Ángeles (2008). Metodologías activas, Grupo de Innovación en Metodologías Activas (GIMA). Valencia: Editorial de la UPV.

Marcelo García, Carlos (1996): Innovación educativa, asesoramiento y desarrollo profesional. Madrid: CIDE.

Martín, Antonio (1992). Ideas prácticas para innovadores críticos. Sevilla: Diada Editores.

Mernissi, Fatima (2002). El harén político. El Profeta y las mujeres. Madrid: Ediciones del Oriente y del Mediterráneo.

Miguel Díaz, Mario De (Coord.) (2006). Metodologías de enseñanza y aprendizaje para el desarrollo de competencias. Orientaciones para el profesorado universitario ente el Espacio Europeo de Educación Superior. Madrid: Alianza.

Parcerisa Aran, Artur (Coord.) (2010). Ejes para la mejora docente en la universidad. Barcelona: Octaedro.

Ovejero Bernal, Anastasio (1990). El aprendizaje cooperativo: una alternativa eficaz a la enseñanza tradicional. Barcelona: PPU.

Ribas Navarro, Manuel (2000). Innovación educativa: teoría, procesos y estrategias. Madrid: Síntesis.

Taylor Mill, Harriet (2000): La emancipación de la mujer. En Ensayos sobre la igualdad de los sexos. Madrid: A. Machado, pp. 113-145. 